\title{
Avaliação da Incorporação de Resíduo de Poli(Etileno- co-Acetato de Vinila) em Borracha Nitrílica
}

\author{
Viviane X. Moreira, Bluma G. Soares \\ Instituto de Macromoléculas, UFRJ
}

\begin{abstract}
Resumo: A influência do resíduo de poli(etileno-co-acetato de vinila) (EVAR) no desempenho mecânico de composições vulcanizadas de NBR foi estudada. A concentração ótima de EVAR, a qual apresentou maior valor de tensão na ruptura, foi de 50 phr. Além disso, a influência do EVA e do EVAR funcionalizado com grupos tióis (EVALSH) e do EVAR modificado com anidrido maleico (EVARCO) como agentes compatibilizantes foi estudada. O copolímero EVALSH atuou como acelerador no processo de vulcanização, sendo observado um decréscimo do tempo de vulcanização. Uma melhora na resistência ao envelhecimento também foi observada. A adição de 10 phr de EVARCO resultou na redução do tempo de vulcanização e em uma melhora na maior parte das propriedades avaliadas. As propriedades termodinâmico-mecânicas foram avaliadas por análises de DMTA. Os estudos da interação entre a fase dispersa e a fase matriz foram realizados utilizando-se microscopia eletrônica de varredura (MEV).
\end{abstract}

Palavras-chave: Composições elastoméricas, resíduo de EVA, propriedades mecânicas, compatibilização.

\section{Evaluation of Waste Poly(ethylene-co-vinyl) Incorporation in Nitrile Rubber}

Abstract: The influence of poly(ethylene-co-vinyl acetate) (EVA) waste (EVAR) on the mechanical performance of NBR vulcanisates compounds was studied. The optimum concentration of EVAR, which has presented higher ultimate tensile stress, was found at $50 \mathrm{phr}$. In addition, the influence of EVA and EVAR functionalized with mercaptan groups (EVALSH) and EVAR functionalized with maleic anidride (EVARCO) as compatibilizing agents was studied. The copolymer EVALSH acted as accelerator for the curing system, since a decreasing of the curing time was observed. An improvement of ageing resistance was also observed. The addition of EVARCO resulted in a reduction of the curing time and an improvement of most of evaluated properties. The thermodynamic-mechanic properties were investigated by DMTA analysis. The studies on the interaction between dispersed phase and matrix were performed by scanning electron microscopy (SEM).

Keywords: Elastomers compounds, EVA waste, mechanical properties, compatibilization.

\section{Introdução}

Um dos vários problemas enfrentados pela humanidade à entrada do século XXI é o da utilização dos resíduos e seu gerenciamento. Devido ao fato dos materiais poliméricos não se decomporem facilmente, a disposição de seus resíduos constitui um sério problema ambiental ${ }^{[1]}$.

O poli(etileno-co-acetato de vinila) (EVA) é um material muito interessante com excelente resistência ao ozônio e à ação de intempéries, boa tenacidade a baixas temperaturas, e boas propriedades mecânicas ${ }^{[2]}$. Outras propriedades específicas, tais como cristalinidade e flexibilidade, são também alcançadas variando-se a quantidade de acetato de vinila no copolímero. Devido à flexibilidade e baixo custo, este copolímero vem sendo bastante utilizado na fabricação de vários artefatos. Uma das mais importantes aplicações de EVA é na indústria calçadista, para fabricação de solados. Devido à grande utilização desse copolímero, uma quantidade considerável de resíduo de EVA (EVAR) é produzida durante os processos de produção das indústrias

Autor para correspondência: Bluma G. Soares, IMA/UFRJ, Caixa Postal: 68525, CEP: 21945-970, Rio de Janeiro, RJ. E-mail: bluma@ima.ufrj.br 
calçadistas do Sul do país. A preocupação da sociedade com o desenvolvimento sustentável, faz com que seja cada vez mais freqüente a busca por métodos de reutilização de resíduos industrias.

Diversas borrachas têm sido misturadas com EVA incluindo borracha natura ${ }^{[3,4]}$, policloropreno ${ }^{[5-8]}$, etc. Misturas de borracha nitrílica (NBR) com EVA também encontram-se relatadas na literatura ${ }^{[2,9]}$. Estas misturas podem constituir uma importante classe de materiais com excelente resistência à óleo, à abrasão e boas propriedades mecânicas promovidas pela fase NBR e excelente resistência ao ozônio e ao oxigênio por causa da fase EVA. De acordo com dados morfológicos relatados na literatura ${ }^{[2]}$, estas misturas são imiscíveis. A imiscibilidade das misturas poliméricas normalmente resulta propriedades mecânicas inferiores devido à baixa adesão interfacial. Este problema pode ser minimizado através do controle da morfologia das fases durante o processamento ou pela adição de agentes compatibilizantes ${ }^{[10]}$.

Em virtude da atual preocupação ambiental e das propriedades apresentadas pelas misturas NBR/EVA, o objetivo do presente trabalho é avaliar a viabilidade da utilização do EVA reciclado, proveniente das indústrias calçadistas em composições de NBR. Além disso, com o objetivo de se encontrar um composto que agisse como um eficiente agente interfacial nas misturas NBR/EVAR, o copolímero EVA e o material reciclado foram modificados com grupos tióis (EVALSH) e com anidrido maleico (EVARCO), respectivamente. As propriedades termodinâmico-mecânicas foram estudadas por DMTA e a interação entre a fase dispersa e a fase matriz foram realizados através de microscopia eletrônica de varredura (MEV).

\section{Experimental}

\section{Materiais}

Copolímero de butadieno - acrilonitrila (NBR) $(\mathrm{N}-615 \mathrm{~B})$; densidade $=0,918 \mathrm{~g} / \mathrm{cm}^{3}$; teor de acrilonitrila combinado igual a $33 \%$ e viscosidade Mooney 48 foi gentilmente cedido pela Nitriflex.

Resíduo de poli(etileno-co-acetato de vinila (EVAR); teor de acetato de vinila igual a $18,3 \%$; teor de gel $=63 \%$ e densidade $=0,995 \mathrm{~g} / \mathrm{cm}^{3}$ foi gentilmente cedido pelas indústrias calçadistas de Caxias do Sul.

Poli(etileno-co-acetato de vinila) (EVA); teor de acetato de vinila igual a $18 \%$ e densidade $=0,937$ $\mathrm{g} / \mathrm{cm}^{3}$ foi gentilmente cedido pela Petroquímica Triunfo S.A

\section{Métodos}

\section{Funcionalização dos copolímeros}

A modificação do EVA virgem com grupos $\mathrm{SH}$ (EVALSH) foi realizada em duas etapas (hidrólise total do EVA e posterior esterificação com ácido mercapto-acético) de acordo com método exposto na literatura ${ }^{[11]}$. A funcionalização do EVAR com anidrido maleico consistiu em duas etapas como está apresentado na Figura 1. A primeira etapa consiste na hidrólise do copolímero reciclado utilizando-se solução metanólica de hidróxido de sódio $(\mathrm{NaOH})$. Ao final do tempo reacional adiciona-se solução $0,1 \mathrm{~N}$ de HCL para neutralizar o $\mathrm{NaOH}$ não reagido. A segunda etapa consiste na reação do EVAR hidrolisado com anidrido maleico, resultando em um copolímero carboxilado, EVARCO, com um grupo insaturado.
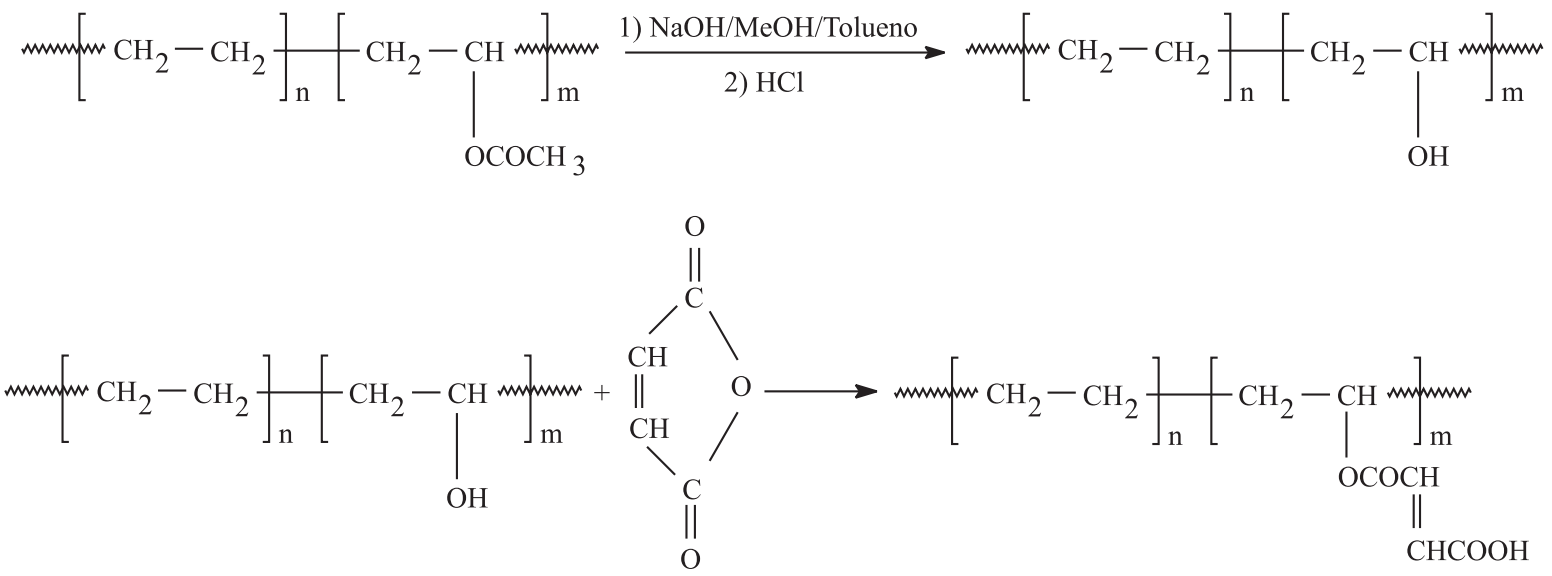

Figura 1. Modificação do copolímero EVAR com anidrido maleico. 
Tabela 1. Concentrações dos materiais empregados nas formulações e tempo de mistura

\begin{tabular}{ccc}
\hline Materiais & $\begin{array}{c}\text { Quantidade } \\
(\mathbf{p h r})^{(\mathbf{a})}\end{array}$ & $\begin{array}{c}\text { Tempo de adição } \\
(\mathbf{m i n})\end{array}$ \\
\hline NBR & 100 & 3 \\
EVALSH/EVARCO & $5 / 10$ & 2 \\
EVAR & Variável & 2 \\
Óxido de zinco & 5,0 & 2 \\
Ácido esteárico & 0,5 & 2 \\
Enxofre & 0,3 & 2 \\
MBTS (b) & 2,0 & 2 \\
TMTD (c) & 1,0 & \\
\hline
\end{tabular}

(a) Partes por cada 100 partes de resina

(b) 2,2-dissulfeto de mercaptobenzotiazila

(c) Dissulfeto de tetrametiltiuram

\section{Preparação das misturas}

Foram preparadas misturas variando-se a concentração de material residual sem copolímero funcionalizado, com 5 phr de EVALSH e com 10 phr de EVARCO. As formulações empregadas encontramse na Tabela 1. As composições foram preparadas em misturador de rolos tipo Berstoff à temperatura de $110^{\circ} \mathrm{C}$ e velocidades dos rolos de 20 e $22 \mathrm{rpm}$.

\section{Determinação dos parâmetros de vulcanização}

A determinação dos parâmetros de vulcanização foi realizada em reômetro de disco oscilatório, à temperatura de $160{ }^{\circ} \mathrm{C}$, arco $1^{\circ}$, durante 24 minutos, segundo norma ASTM D2084 - 81, usando Curômetro TI - 100 Tecnologia Industrial.

\section{Elaboração dos corpos de prova para ensaios}

Para obtenção dos corpos de prova do ensaio de resistência à tração e envelhecimento foram moldadas placas de dimensões $100 \times 90 \times 2 \mathrm{~mm}$ por compressão em prensa hidráulica à temperatura de $160{ }^{\circ} \mathrm{C}$ no tempo ótimo de cura (fornecido pelo reômetro), sob pressão de 15000 libras. Os corpos de prova foram cortados conforme técnica DIN 53504, a partir das placas prensadas.

Os corpos de prova para análise termodinâmico mecânica foram cortados dos tapetes prensados nas dimensões 10x5x2 mm.

\section{Propriedades mecânicas}

Os resultados de módulo a 300\%, tensão e alongamentos na ruptura foram obtidos em máquina de ensaio universal Instron, modelo 1101, TM-M, com célula de carga $100 \mathrm{Kg}$ e velocidade das garras de $200 \mathrm{~mm} / \mathrm{min}$.

\section{Ensaio de envelhecimento}

Os corpos de prova cortados para o ensaio de resistência à tração, foram colocados em estufa de circulação de ar, à temperatura de $70{ }^{\circ} \mathrm{C}$, durante 72 horas, segundo a norma ASTM D573-81.

\section{Propriedades termodinâmico-mecânicas (DMTA)}

As propriedades termodinâmico mecânicas das composições estudadas foram determinadas usando DMTA, Rheometric MK III, na faixa de temperatura de -80 a $20{ }^{\circ} \mathrm{C}$, velocidade de aquecimento de $2{ }^{\circ} \mathrm{C} / \mathrm{min}$ e a freqüência de $10 \mathrm{~Hz}$.

\section{Microscopia eletrônica de varredura (MEV)}

A amostras para microscopia foram fraturadas em nitrogênio líquido e as superfícies de fratura metalizadas com ouro em metalizador JEOL IFC 15000. As análises morfológicas foram realizadas em microscópio eletrônico de varredura ZEISS, modelo DSM 960.

\section{Deformação permanente à compressão}

O método utilizado para determinação da resistência à compressão seguiu a norma ASTM D395-85. As amostras foram colocadas em estufa de circulação forçada de ar, à temperatura de $70^{\circ} \mathrm{C}$, por um período de 22 horas.

\section{Resultados e Discussão}

\section{Misturas NBR/EVAR}

\section{Parâmetros de vulcanização, propriedades} mecânicas e resistência ao envelhecimento

A Tabela 2 apresenta os parâmetros de vulcanização, torque mínimo (Ml), torque máximo $(\mathrm{Mh})$, tempo ótimo de cura $\left(\mathrm{t}_{90}\right)$, o tempo de pré-vulcanização $\left(t_{s 1}\right)$, obtidos a partir do reômetro de disco oscilatório, bem como as propriedades mecânicas e a avaliação da resistência ao envelhecimento, correspondentes às misturas estudadas.

Quanto aos parâmetros de vulcanização, pode-se observar que o torque mínimo aumenta à medida que 
o teor de EVAR aumenta até a concentração de 70 phr e todas as composições apresentaram maiores valores de torque mínimo do que a goma pura. O aumento no torque mínimo está associado a um aumento na viscosidade do sistema ${ }^{[12,13]}$. Como o EVA reciclado é um material reticulado, já era esperado que um aumento de sua quantidade na composição provocasse um acréscimo na viscosidade. Curiosamente, a mistura com 90 phr de EVA reciclado apresentou um decréscimo no torque mínimo. Visto que, este copolímero é um material reticulado com teor de gel de $63 \%$, sugere-se que nesta concentração a fração solúvel de EVA, que corresponde a 37, esteja influenciando de forma a reduzir a viscosidade do sistema, visto que o EVA pode agir como um auxiliar de processamento ${ }^{[14]}$.

Quanto ao torque máximo, que é uma medida do grau de reticulação, observa-se que de uma maneira geral, a presença de uma maior quantidade de material reciclado na mistura não alterou de modo significativo os valores deste parâmetro até $70 \mathrm{phr}$. No entanto, observou-se um decréscimo para a mistura com 90 phr. Este comportamento pode estar associado a um decréscimo relativo da fase NBR, uma vez que somente esta fase pode sofrer reticulação no sistema com enxofre.

Quanto ao tempo de pré-vulcanização $\left(t_{\mathrm{s} 1}\right)$ e tempo ótimo de vulcanização $\left(t_{90}\right)$, a adição de material reciclado provocou um aumento em ambos os parâmetros. A adição do EVAR contribui para uma reação de vulcanização mais lenta até 30 phr quando comparado à goma pura. $\mathrm{O}$ uso do EVAR na mistura apresenta uma vantagem no que diz respeito ao tempo de pré-vulcanização. Este parâmetro é uma das variáveis críticas relacionadas ao processo de vulcanização. Composições com valores mais altos de tempo de prévulcanização apresentam, conseqüentemente, uma maior resistência à pré-vulcanização ${ }^{[12,14,15]}$. A importância da resistência à pré-vulcanização está no fato de que o processamento requer tempos consideráveis a elevadas temperaturas, permitindo uma maior segurança durante as etapas de processamento e estocagem do material.

Tabela 2. Propriedades das misturas NBR/EVAR

\begin{tabular}{|c|c|c|c|c|c|}
\hline \multirow{2}{*}{ Propriedades } & \multicolumn{5}{|c|}{$\begin{array}{c}\text { Teor de EVAR } \\
\text { (phr) }\end{array}$} \\
\hline & $\mathbf{0}$ & 20 & 50 & 70 & 90 \\
\hline Ml (lb.in) & 2,5 & 3,3 & 4,0 & 5,1 & 4,2 \\
\hline Mh (lb.in) & 15,8 & 16,7 & 15,7 & 16,6 & 10,0 \\
\hline$t_{90}(\min )$ & 7,0 & 7,7 & 9,5 & 9,5 & 15,1 \\
\hline $\mathrm{t}_{\mathrm{s} 1}(\mathrm{~min})$ & 2,8 & 4,2 & 5,0 & 5,2 & 8,6 \\
\hline Tensão na ruptura $(\mathrm{MPa})$ & $2,4 \pm 0,3$ & $3,3 \pm 0,5$ & $4,7 \pm 0,5$ & $3,6 \pm 0,7$ & $3,5 \pm 0,3$ \\
\hline Alongamento na ruptura (\%) & $625 \pm 76$ & $600 \pm 68$ & $575 \pm 63$ & $475 \pm 65$ & $500 \pm 56$ \\
\hline Módulo a 300 \% (MPa) & $1,3 \pm 0,3$ & $1,9 \pm 0,1$ & $2,8 \pm 0,2$ & $3,4 \pm 0,4$ & $3,8 \pm 0,3$ \\
\hline $\begin{array}{l}\text { Deformação permanente à } \\
\text { compressão }(\%)\end{array}$ & 39,6 & 42,9 & 56,2 & 62,3 & 67,6 \\
\hline
\end{tabular}

Propriedade após o envelhecimento

\begin{tabular}{l|ccccc}
\hline Módulo a $300 \%(\mathrm{MPa})$ & $0,8 \pm 0,2$ & $1,3 \pm 0,2$ & $2,4 \pm 0,3$ & $2,5 \pm 0,2$ & $2,7 \pm 0,2$ \\
$\Delta \sigma_{1}(\%)$ & -33 & -32 & -14 & -26 & -29 \\
Tensão na ruptura (MPa) & $1,6 \pm 0,5$ & $2,9 \pm 0,2$ & $3,7 \pm 0,3$ & $3,3 \pm 0,3$ & $2,9 \pm 0,3$ \\
$\Delta \sigma_{2}(\%)$ & -38 & -12 & -21 & $-8,0$ & -17 \\
Alongamento na ruptura (\%) & $350 \pm 31$ & $500 \pm 19$ & $575 \pm 63$ & $400 \pm 32$ & $400 \pm 27$ \\
$\Delta \varepsilon(\%)$ & -44 & -17 & -30 & -26 & -20 \\
\hline
\end{tabular}


Variando-se a concentração de EVAR nas composições de NBR estudadas, observa-se que todas as misturas apresentaram valores superiores de tensão na ruptura quando comparados à goma pura. Pode-se observar ainda, que o valor da tensão na ruptura aumenta à medida que a concentração de material reciclado é aumentada na mistura até o teor de 50 phr. A partir de $70 \mathrm{phr}$, pode-se verificar uma redução da tensão na ruptura. Isto sugere que o teor ótimo de material reciclado, até o qual é possível se observar um aumento na tensão na ruptura é 50 phr. Provavelmente, até $50 \mathrm{phr}$ a dispersão do material reciclado moído na matriz elastomérica é favorecida, garantindo uma boa interação entre as fases ${ }^{[12,16]}$.

Quanto ao alongamento na ruptura não foram observadas diferenças significativas entre os valores das composições com EVAR e da goma pura. É observado um ligeiro decréscimo na deformação na ruptura para as misturas a partir de $70 \mathrm{phr}$ de EVAR.

O módulo a $300 \%$ aumenta com o acréscimo de material reciclado na mistura provavelmente porque o aumento do número de partículas de material reticulado introduzidas na matriz elastomérica reduz a elasticidade das cadeias tornando o material mais rígido.

Quanto à resistência à compressão, verifica-se que o aumento da concentração de material reciclado na mistura aumenta a deformação permanente à compressão reduzindo, desta forma, a resistência das misturas quando comparadas à goma pura. Sugere-se que a baixa resistência à compressão esteja associada à fase EVA que possui baixa elasticidade.

As percentagens de retenção das propriedades módulo a $300 \%$, tensão e alongamento na ruptura, após envelhecimento em estufa de circulação forçada de ar estão representadas por $\Delta \sigma_{1}, \Delta \sigma_{2} \mathrm{e} \Delta \varepsilon$, respectivamente. Em relação às propriedades após o envelhecimento, observa-se que todas as composições apresentaram redução da propriedade em função da degradação sofrida durante o processo de envelhecimento. Contudo, as misturas com EVAR apresentaram maior resistência ao envelhecimento do que a borracha pura provavelmente devido à ausência de insaturações na cadeia do copolímero EVAR.

\section{Análise morfológica}

O estudo da homogeneidade e da dispersão entre as fases das misturas foi realizado empregando-se microscopia eletrônica de varredura (MEV).
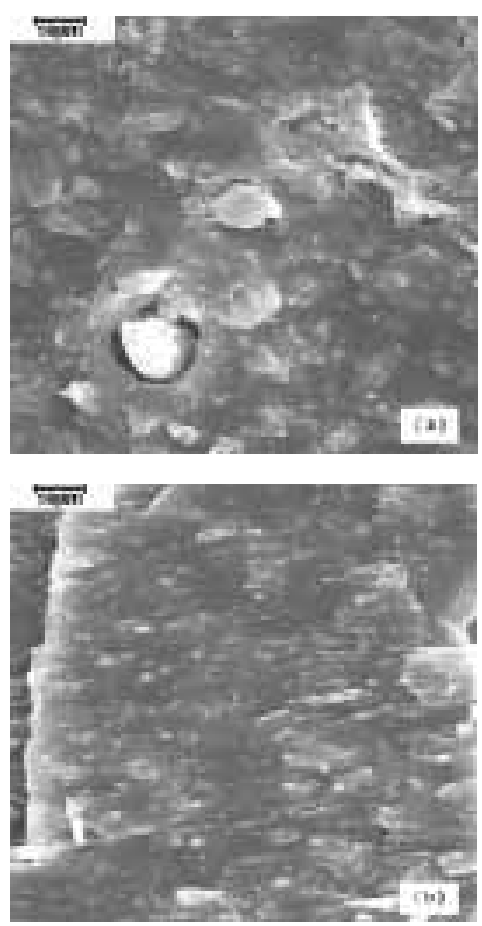

Figura 2. Micrografias obtidas por microscopia eletrônica de varredura das misturas NBR/EVAR: (a) $20 \mathrm{phr}$ e (b) $50 \mathrm{phr}$.

A Figura 2 apresenta as micrografias das misturas NBR/EVAR contendo 20 e $50 \mathrm{phr}$ de EVAR. Podese observar na composição com 20 phr de EVAR (Figura 2a), a heterogeneidade da mistura e domínios razoavelmente grandes da fase dispersa. Em relação à composição NBR/EVAR com 50 phr de EVA reciclado (Figura 2b), observa-se uma maior homogeneidade no sistema. $\mathrm{O}$ aumento da quantidade de resíduo implica em um acréscimo na viscosidade do sistema expresso em termos dos valores de torque mínimo, o que requer uma maior força cisalhante para a preparação da mistura. Esta força, provavelmente, deve ter contribuído para a quebra dos aglomerados de EVAR, provocando a redução dos domínios. Esta morfologia pode ter sido responsável pela melhora nas propriedades de tensão.

\section{Propriedades termodinâmico-mecânicas}

Os resultados das propriedades termodinâmicomecânicas estão apresentados em termos do módulo de estocagem (E') e da tangente de perda $(\tan \delta)$. A Figura 3 mostra as propriedades das composições vulcanizadas de NBR incorporadas com diferentes teores do resíduo, em função da temperatura. $\mathrm{O}$ valor de E' aumenta com o teor de resíduo e a composição com 50 phr é a que apresenta maior valor de módulo. Estes resultados podem ser reforçados considerando 


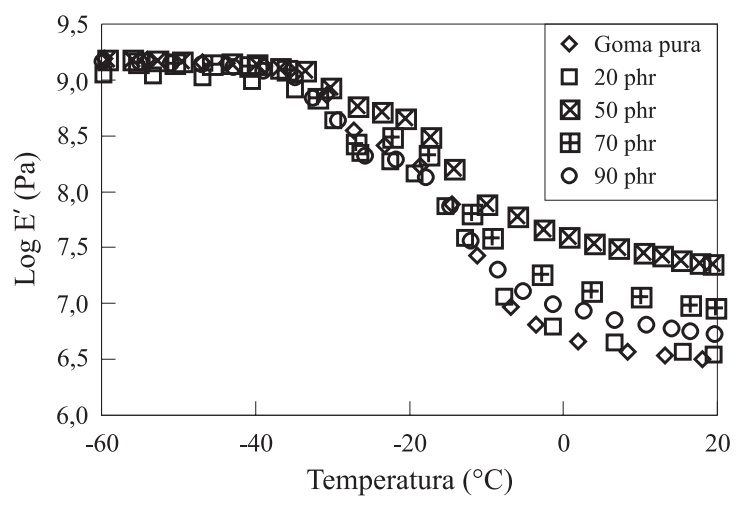

Figura 3. Módulo de estocagem em função da temperatura para as diferentes composições de NBR.

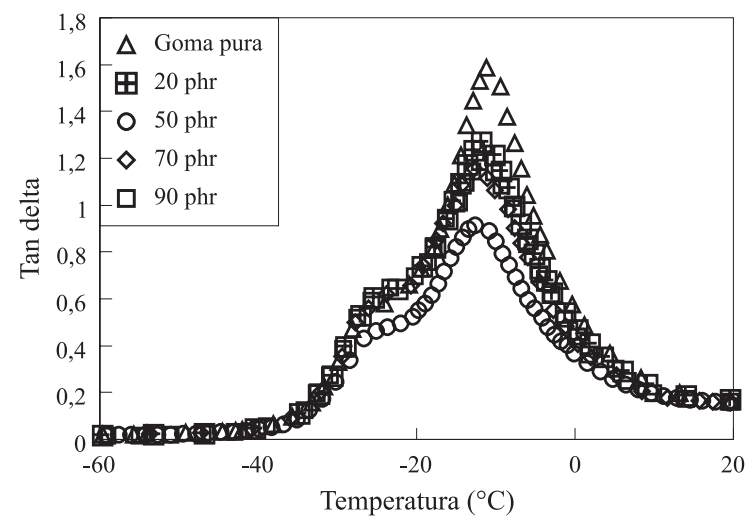

Figura 4. Tangente de perda em função da temperatura para as diferentes composições de NBR.

a tangente de perda $(\tan \delta)$. No caso da $\tan \delta$, os valores máximos $\left(\tan \delta_{\max }\right.$ ) foram observados em torno da temperatura de transição. Um aumento no valor da $\tan \delta_{\max }$ implica numa perda de resistência mecânica do sistema ${ }^{[17]}$. Estas perdas estão relacionadas com a maior energia requerida para a movimentação das cadeias poliméricas próximo à região de transição ${ }^{[17]}$. Assim, pode-se observar na Figura 4 que o menor valor de $\tan \delta_{\max }$ encontrado foi para a mistura com 50 phr de EVA reciclado sugerindo uma menor mobilidade das cadeias poliméricas com este teor de resíduo. Para concentrações superiores de resíduo, os valores de $\tan \delta_{\max }$ aumentam novamente.

\section{Misturas com os copolímeros funcionalizados}

\section{Parâmetros de vulcanização, propriedades mecânicas e resistência ao envelhecimento}

Foram preparadas três diferentes misturas, a fim de se estudar a possibilidade de se utilizar um agente compatibilizante eficiente para as misturas NBR/ EVAR. Os copolímeros EVALSH e EVARCO foram empregados nas misturas NBR/EVAR e suas proprie- dades comparadas à mistura sem copolímero funcionalizado. As propriedades avaliadas bem como as composições das misturas estudadas, estão apresentadas na Tabela 3.

Quanto ao emprego do copolímero funcionalizado com grupos tióis, de uma maneira geral, estes grupos reagem facilmente com duplas ligações e são largamente empregados na funcionalização de elastômeros. Desta forma, tais grupos poderiam reagir quimicamente com a fase elastomérica mais insaturada durante o processamento. Como a cadeia polimérica do EVALSH apresenta estrutura química semelhante à da fase EVA, acredita-se que o EVALSH age como agente interfacial entre as fases ${ }^{[18]}$.

Quanto aos parâmetros de cura da mistura com EVALSH, observa-se que a presença de EVALSH nas misturas não provocou alterações significativas nos valores de torque mínimo, torque máximo ao serem comparados com os resultados destes parâmetros encontrados para as misturas NBR/EVAR. Quanto ao tempo de pré-vulcanização e tempo ótimo de cura, observa-se na Tabela 3, que a adição do EVALSH contribuiu para a redução destes parâmetros em relação à mistura sem EVALSH. O EVALSH, então, agiu no processo de cura acelerando a reação de vulcanização. De fato, De fato, grupos tióis podem atuar como aceleradores, citando como exemplo, o acelerador de dissulfeto de bis(diisopropil)tiofosforila, utilizado também como agente interfacial de misturas envolvendo copolímero de butadieno-estireno (SBR) e borracha nitrílica carboxilada (XNBR $)^{[18]}$.

Quanto á resistência à tração, a mistura com EVALSH não apresentou variação significativa quanto ao valor de tensão na ruptura encontrado, comparando-se ao resultado apresentado pela mistura NBR/ EVAR sem copolímero modificado. Provavelmente, o fato do material reciclado ser reticulado, não contribuiu para uma boa afinidade entre a fase constituída pelo EVALSH, sintetizado a partir do EVA virgem, e a fase constituída pelo EVAR. Desta forma, o EVALSH não agiu como agente interfacial quanto à resistência à tração.

Quanto à deformação na ruptura, também não foram observadas diferenças significativas com a adição de EVALSH na mistura.

Em relação ao módulo a $300 \%$, a mistura com EVALSH apresentou um aumento discreto quando comparado ao resultado encontrado para a mistura sem EVALSH. Esta melhora no módulo a 300 \% pode 
Tabela 3. Estudo da ação dos copolímeros EVALSH e EVARCO nas propriedades das misturas NBR/EVAR

\begin{tabular}{|c|c|c|c|}
\hline \multirow{2}{*}{ Propriedade } & \multicolumn{3}{|c|}{$\begin{array}{c}\text { Composição } \\
\text { (phr) }\end{array}$} \\
\hline & EVAR 100/20 & $\begin{array}{c}\text { EVAR/EVALSH } \\
(20 / 5)\end{array}$ & $\begin{array}{c}\text { EVAR/EVARCO } \\
(10 / 10)\end{array}$ \\
\hline Ml (lb.in) & 3,3 & 3,8 & 8,6 \\
\hline Mh (lb.in) & 16,7 & 15,3 & 26,8 \\
\hline $\mathrm{t}_{90}(\mathrm{~min})$ & 7,7 & 6,3 & 5,8 \\
\hline $\mathrm{t}_{\mathrm{s} 1}(\min )$ & 4,2 & 3,1 & 3,7 \\
\hline Tensão na ruptura (MPa) & $3,3 \pm 0,5$ & $3,6 \pm 0,5$ & $4,9 \pm 0,3$ \\
\hline Alongamento na ruptura (\%) & $600 \pm 68$ & $675 \pm 37$ & $725 \pm 20$ \\
\hline Módulo a 300 \% (MPa) & $1,9 \pm 0,1$ & $2,0 \pm 0,3$ & $2,9 \pm 0,1$ \\
\hline Deformação permanente à compressão (\%) & 42,9 & 41,2 & 27,2 \\
\hline \multicolumn{4}{|c|}{ Propriedade após o envelhecimento } \\
\hline Módulo a 300 \% (MPa) & $1,3 \pm 0,2$ & $2,6 \pm 0,3$ & $1,7 \pm 0,4$ \\
\hline$\Delta \sigma_{1}(\%)$ & -32 & +30 & -41 \\
\hline Tensão na ruptura (MPa) & $2,9 \pm 0,2$ & $4,0 \pm 0,4$ & $2,8 \pm 0,4$ \\
\hline$\Delta \sigma_{2}(\%)$ & -12 & +11 & -43 \\
\hline Alongamento na ruptura (\%) & $500 \pm 19$ & $575 \pm 36$ & $400 \pm 24$ \\
\hline$\Delta \varepsilon(\%)$ & -17 & -17 & -45 \\
\hline
\end{tabular}

estar relacionada às interações entre os grupos mercaptan e as ligações duplas da matriz nitrílica.

Em relação à resistência à compressão, não foi observada nenhuma variação nesta propriedade em função da adição do EVALSH na mistura.

Quanto à resistência ao envelhecimento, pode-se observar que os valores de módulo a 300 \% e tensão na ruptura foram superiores após a degradação. Sugere-se que este acréscimo seja devido à ocorrência de póscura durante o ensaio de envelhecimento.

Com o objetivo de desenvolver um composto que atuasse de maneira eficiente como agente interfacial nas misturas NBR/EVAR, o EVA reciclado foi funcionalizado com grupos carboxílicos conforme está descrito na parte experimental. A mistura foi preparada utilizando-se $10 \mathrm{phr}$ do copolímero reciclado funcionalizado e mesmo teor de material reciclado.

Em relação aos parâmetros de vulcanização, a mistura com EVARCO apresentou valor de torque mínimo superior à mistura NBR/EVAR e à mistura NBR/EVAR com 5 phr de EVALSH. Isto sugere uma maior interação entre as fases, acarretando um aumento na viscosidade do sistema.

Quanto ao torque máximo, observa-se um maior valor para a mistura com EVARCO. Sugere-se que nesta proporção, esteja havendo uma maior interação entre as fases e que o EVARCO esteja contribuindo para um maior grau de reticulação.

Quanto ao tempo de pré-vulcanização e ao tempo ótimo de vulcanização, os resultados para a mistura com EVARCO não apresentaram variações significativas quanto aos valores encontrados para a mistura com EVALSH. O tempo de pré-vulcanização, apesar de ser menor do que o valor encontrado para a mistura NBR/EVAR sem agente compatibilizante, foi superior ao resultado medido para a goma pura e apresentado na Tabela 2. $\left(\mathrm{t}_{\mathrm{s} 1}=2,8 \mathrm{~min}\right)$, contribuindo, assim, para a minimização do risco de ocorrência de pré- vulcanização. A mistura EVAR/EVARCO (10:10) apresentou valor de tempo ótimo de vulcanização inferior ao da goma pura $\left(t_{90}=7,0 \mathrm{~min}\right)$, sugerindo a ação do EVARCO na reação de vulcanização, tornando-a mais rápida. Sugere-se que ocorra 
neste sistema o fenômeno já descrito na literatura ${ }^{[18]}$, em que o óxido de zinco do sistema ativador reage com a carboxila do resíduo funcionalizado. Esta reação faz com que o sistema ativador, cuja dispersão é mais favorecida na matriz nitrílica, esteja incorporado no resíduo e não apenas disperso no sistema. Provavelmente, isto faz com que além de se promover uma maior interação entre as fases, ocorra uma diminuição no tempo ótimo de cura.

Quanto as valores de tensão na ruptura, observa-se um valor superior para a mistura com EVARCO em relação às misturas NBR/EVAR e NBR/EVAR com 5 phr de EVALSH. A melhora nas propriedades de tensão atingida pelo EVARCO pode ser explicada pela localização do grupo reativo, que se encontra na superfície do EVA reciclado. No caso do EVALSH, existem 3 fases distintas - NBR (matriz), EVA reciclado e EVALSH não reticulado, que apesar de reagir quimicamente com as duplas ligações da NBR, pode ou não estar interagindo com os domínios do EVAR. Além disso, o fato do óxido de zinco poder estar reagindo com a carboxila no resíduo funcionalizado, provavelmente está contribuindo para o aumento da interação entre as fases.

Quanto à deformação na ruptura, a misturas com EVARCO apresentou um discreto aumento em relação às demais misturas estudadas.

Quanto ao módulo a 300\% e à resistência à compressão, observa-se os melhores resultados para a mistura utilizando resíduo de EVA funcionalizado com grupos carboxílicos maleico. A maior resistência à compressão está expressa em termos da menor deformação permanente à compressão (DCP). É importante salientar que esta mistura também apresentou menor deformação permanente à compressão do que a goma pura $(\mathrm{DCP}=39,6 \%)$. Apesar da mistura empregando material reciclado funcionalizado ter apresentado o melhor desempenho quanto às propriedades avaliadas, quanto à resistência ao envelhecimento os resultados não foram satisfatórios. Pode-se observar que todas as propriedades sofreram um decréscimo em função da degradação. Além disso, a mistura com EVARCO apresentou resistência ao envelhecimento, expressa em termos da menor retenção de propriedades, inferior à mistura sem o agente compatibilizante e em relação às demais composições estudadas. Isto se deve provavelmente ao fato de que quando se realiza a reação entre o EVAR e o anidrido maleico, apresentada na Figura 1, são introduzidos mais grupos insaturados na cadeia do polímero. Esta maior quantidade de insaturações deve ser o principal responsável pela redução da resistência ao envelhecimento das misturas contendo EVA reciclado funcionalizado.

\section{Análise morfológica}

São apresentadas a seguir as microscopias das misturas EVAR/EVALSH (20/5) e EVAR/EVARCO (10:10) na Figura 5. A microscopia da composição com 20 phr do resíduo sem a adição de qualquer copolímero modificado já foi apresentada anteriormente na Figura 2a. Apesar de não ter sido observada uma melhora quanto às propriedades de tensão, a mistura com 5 phr de EVALSH (Figura 5a) apresentou maior homogeneidade e menores domínios da fase dispersa na matriz, se comparada à mistura com 20 phr de EVAR sem a adição de EVALSH (Figura 2a).

A morfologia da mistura EVAR/EVARCO (10:10) (Figura 5b), apresentou-se mais homogênea com menores domínios da fase dispersa se comparada às demais misturas. Esta morfologia pode ter contribuído para a melhor propriedade de tensão encontrada para esta mistura.

Como para todas as misturas a quantidade de EVA residual é a mesma, ou seja, $20 \mathrm{phr}$, a mistura com
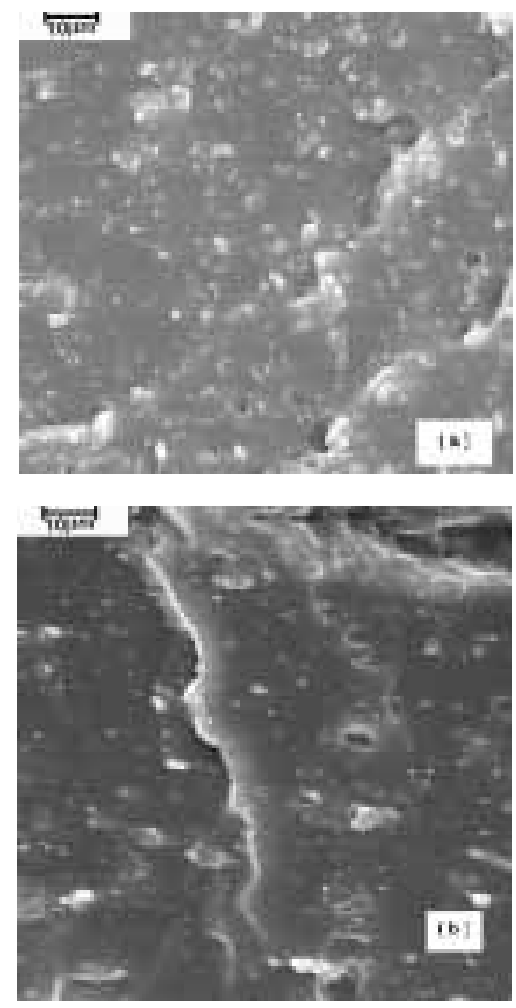

Figura 5. Micrografias obtidas por microscopia eletrônica de varredura das misturas: (a) EVAR/EVALSH (20:5) phr e (b) EVAR/EVARCO $(10: 10) \mathrm{phr}$. 


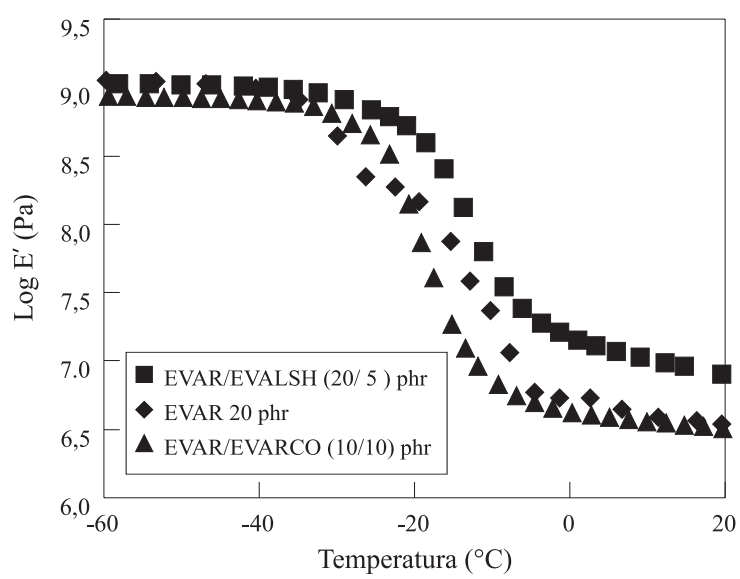

Figura 6. Módulo de estocagem em função da temperatura variando-se o agente interfacial.

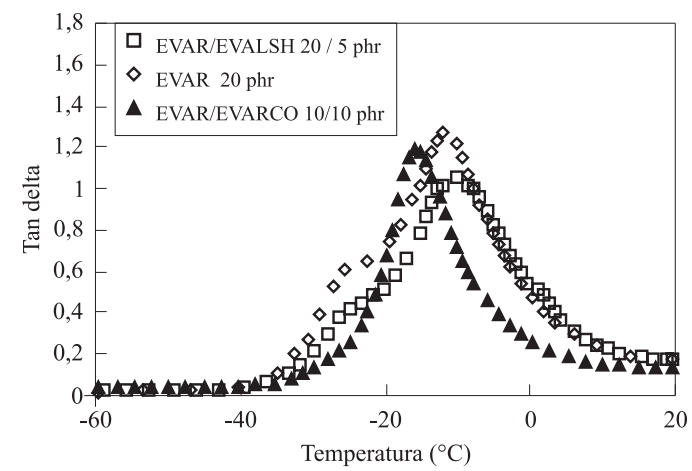

Figura 7. Variação da tangente de perda em função da temperatura variando-se o agente interfacial.

EVARCO, apresentou a morfologia mais dispersa, como pode ser verificada na Figura 5b. Esta maior dispersão, considerando a mesma percentagem mássica de material adicionado à matriz e o mesmo volume de NBR, pode estar acarretando um aumento no volume livre, permitindo o aumento da mobilidade molecular, verificado no aumento da $\tan \delta_{\max } \mathrm{e}$ na redução da $\mathrm{Tg}$ para temperaturas inferiores.

\section{Propriedades termodinâmico-mecânicas}

A Figura 6 apresenta a variação do módulo de estocagem (E') em função da temperatura comparando as composições NBR/EVAR com 20 phr do resíduo, a mistura com $20 \mathrm{phr}$ de EVAR e $5 \mathrm{phr}$ de EVALSH, e ainda, a mistura com 10 phr de EVAR e 10 phr de EVARCO. Pode-se observar que o módulo da composição com EVALSH foi superior aos valores encontrados para as demais misturas. A Figura 7 apresenta o comportamento da tangente de perda ( $\tan \delta$ ) em função da temperatura, comparando as composições com e sem copolímeros funcionalizados.

Pode-se observar que o menor valor de $\tan \delta_{\max }$ foi verificado para a composição com EVALSH, indicando uma maior restrição do movimento molecular das cadeias de NBR neste sistema. A mistura com EVARCO apresentou um maior valor de $\tan \delta_{\max }$, no entanto, observa-se um deslocamento do valor da Tg para região de temperaturas inferiores.

\section{Conclusões}

Com relação aos parâmetros de cura, a presença de EVA reciclado (EVAR) ocasionou um aumento nos valores de torque mínimo com relação à goma pura até a concentração de $70 \mathrm{phr}$, o que foi atribuído ao aumento na viscosidade do sistema, uma vez que EVAR já apresenta um teor de material reticulado.

$A$ incorporação de EVA reciclado ocasionou um aumento no tempo ótimo de cura, no entanto, contribuiu para um decréscimo no tempo de pré-vulcanização, minimizando a ocorrência de pré-cura.

Quanto à viabilidade de se utilizar o material reciclado em composições de NBR visando uma melhoria em suas propriedades, pôde-se verificar que a adição resíduo melhorou as propriedades analisadas quando comparadas aos resultados encontrados para goma pura. O teor ótimo de EVA reciclado encontrado para as composições estudadas foi de $50 \mathrm{phr}$. A deformação permanente à compressão aumenta com a adição de resíduo, o que reflete em uma perda na resistência à compressão.

No caso do EVALSH, não foi observada uma melhora em relação às propriedades de tensão e à deformação permanente à compressão. Neste caso, existem 3 fases distintas - NBR (matriz), EVA reciclado e EVALSH não reticulado, que apesar de reagir quimicamente com as duplas ligações da NBR, pode ou não estar interagindo com os domínios do EVAR. A compatibilização das misturas NBR/EVAR com a adição de EVALSH foi efetiva no que diz respeito ao módulo a $300 \%$. Este aditivo ocasionou também uma melhor homogeneização das misturas entre NBR e EVA reciclado e a redução no tempo ótimo de cura.

Quanto à resistência ao envelhecimento a ausência de insaturações no copolímero EVAR pode estar contribuindo para a maior resistência ao envelhecimento das misturas com material reciclado

$\mathrm{A}$ adição de 10 phr de EVARCO à composição com 10 phr de EVA reciclado contribui para um acréscimo nos valores de torque máximo e uma redução 
significativa nos valores de tempo ótimo de vulcanização e aumentou o tempo de pré-vulcanização em relação à goma pura, o que facilita a aplicação tecnológica desta mistura, pois levaria mais tempo para iniciar a vulcanização, mas o processo de vulcanização é rápido.

A mistura NBR/EVARCO/EVAR 100:10:10 phr apresentou resultados bastante satisfatórios quanto as propriedades mecânicas avaliadas, excetuando-se a resistência ao envelhecimento. Sugere-se que o resíduo funcionalizado apresente uma maior afinidade com a fase EVA reticulado, devido à maior semelhança quanto à estrutura química, o que contribuiu para uma melhora no desempenho da mistura em relação à composição sem a adição de EVARCO.

Sugere-se que o resíduo funcionalizado com grupos carboxila apresenta uma ação compatibilizante mais acentuada devido à boa afinidade com a fase reticulada. Além disso, os grupos carboxila são capazes de reagir com o ativador $\mathrm{ZnO}$ localizado preferencialmente na fase NBR com a formação de ionômero interfacial o que favorece uma maior adesão entre as fases.

\section{Agradecimentos}

Os autores gostariam de agradecer ao CEPG UFRJ, CAPES, CNPQ (Proc $\mathrm{n}^{\circ}$ 130547/2000.0), PADCT (Proc. No 620132/98-1) e à FAPERJ pelo auxílio financeiro.

\section{Referências Bibliográficas}

1. Debapriya, D.; Sukumar, M.; Adhikari, B.- Journal. of Applied Polymer Science, 73, 2951 (1999).

2. Varghese H.; Bhagawan S. S.; Someswara Rao S.; Thomas S.- European Polymer Journal, , 31, 957 (1995).

3. Koshy, A T.; Kuriakose, B.; Thomas, S. - Polym Degrad Stab, 36, 137 (1992).
4. Koshy, A T.; Kuriakose, B.; Thomas, S.; Premaltha, C. K.; Varguese, S. - Journal of Applied Polymer Science, 49, 901 (1993).

5. Kundu, P.P.; Tripathy, D. K - Kautsc Gummi Kunstst, 49, 268 (1996).

6. Kundu, P.P.; Tripathy, D. K; Gupta, B. R.- Journal of Applied Polymer Science, 63, 187 (1997).

7. Kundu, P.P.; Tripathy, D. K - Kautsc Gummi Kunstst, 49, 666 (1996).

8. Kundu, P.P.; Banerjee, S.; Tripathy, D. K - Int Journal Polym Mater, 32, 125 (1996)., 49, 666 (1996).

9. Bandyopadhway, G. G.;Bhagawan, S.S.; Ninan, K.N.; Thomas, S. - Rubber Chem Technol, 70, 650 (1997).

10. Koning, C.; Van Duin, M.; Pagnoule, C.; Jerome, R. Prog Polym Sci , 61, 591 (1996).

11. Dutra, R.C.L.; Lourenço, V.L.; Diniz, M.F.; Azevedo, M.F.P; Barbosa, R.V.; Soares, B. G.- Polym Bulletin, 37, 215 (1996).

12.. Ishiaku U.S.; Chong C.S.; Ismail H.-Polymers \& Polymers Composites, 6, 399 (1998).

13. Kim J. I.; Ryu S. H.; Chang Y. W. - Journal of Apllied Polymer Science, 58, 101 (2000).

14. Kuczkowsky J. A - Encyclopedia of Polymer Science and Engineering, editado por Mark H. F., Bikales N. M., Overberger C.G. e Menges G.; John Wiley \& Sons, New york, vol.17, p. 716.

15. Coran A Y.- Encyclopedia of Polymer Science and Engineering, editado por Mark H. F., Bikales N. M., Overberger C.G. e Menges G.; John Wiley \& Sons, New york, vol.17, p. 666.

16. Boonstra B. B. - Polymer, 20, 691 (1979).

17. Sombatsompop N. - Journal of .Applied Polymer Science, 74, 1129. (1999).

18. Pimblott J. G.; Scott G.; Stuckey E. - Journal of Applied Polymer Science, 69, 865 (1975).

Recebido: 10/08/01

Aprovado: 02/07/02 